\title{
La gestion concertée et décentralisée de l'eau en France
}

\section{Coordinated and decentralised water resources management in France}

\author{
par C. Jousseaume \\ Agence de l'eau Adour-Garonne \\ Y. Retkowsky \\ MATE / Direction de l'eau
}

After first remembering the principal steps that have been used in setting up the French water-management policy (planning, programme elaboration, contractual partnership) and the, showing the particular devices chosen for following up such policy, this contribution, is intended to present a state of the first experiences enforced, and, with their results, to aim at confirming or modifying the devices.

This survey also points out that the concept of an integrated, concerted and decentralized water management is more and more set into current practice at local levels with water development and management plans (SAGE's) and river or bay contracts.

\section{I 口 LES SCHÉMAS DIRECTEURS D'AMÉNAGEMENT ET DE GESTION DES EAUX (SDAGE)}

Ils ont été adoptés par les Comités de Bassin et approuvés par les Préfets coordonnateurs de Bassin au cours du deuxième semestre de 1996. Depuis lors ils encadrent les décisions publiques et les programmes de l'Etat et des collectivités locales qui, dans le domaine de l'eau, devront leur être compatibles (ou rendus compatibles).

Leur élaboration a été l'occasion d'un débat intense, ouvert et fructueux entre tous les partenaires concernés et a montré que des enjeux conflictuels, des négociations difficiles,... peuvent aboutir à un projet fort pour l'avenir de la gestion des eaux. Reste à présent à les mettre en œuvre et à en assurer un suivi à travers des tableaux de bord. Ce suivi permettra d'évaluer l'impact, le bien-fondé ou la faiblesse, des principaux axes de la politique de l'eau, afin de pouvoir les infléchir en temps utile au niveau des bassins et voire même au niveau national.

\subsection{Un tableau de bord national}

Le tableau de bord national, tronc commun des six SDAGE à l'échelle du territoire, a été élaboré par un groupe de travail inter-bassins en s'appuyant sur les premiers projets de tableaux de bord des SDAGE.

Quatre catégories d'indicateurs ont été retenues, traduisant :

- pour les trois premières, la mise en œuvre des principales mesures préconisées par les SDAGE : 1.l'amélioration des connaissances, le dispositif de suivi et les outils d'évaluation,

2.l'organisation de la gestion, la planification et la réglementation,

3. les équipements, les travaux et l'amélioration des pratiques.

- et pour la dernière,

4. leurs effets sur l'évolution de l'état des milieux naturels et de la satisfaction des usages, qui devrait refléter, sauf facteurs extérieurs, les résultats de la bonne exécution de la politique de l'eau.

Elles recouvrent les différents axes de cette politique, tels que déclinés dans les SDAGE autour de six thèmes majeurs :

a) la prévention et la gestion des risques naturels et technologiques,

b) la gestion et la protection des milieux aquatiques,

c) la gestion qualitative de la ressource,

d) l'alimentation en eau potable et la santé publique,

e) la gestion quantitative de la ressource,

f) l'organisation de la gestion concertée.

\subsection{Le renseignement des tableaux de bord}

Le renseignement des tableaux de bord des SDAGE s'appuiera sur deux groupes d'acteurs principaux :

- les services de l'Etat coordonnés par les DIREN de Bassin, notamment dans le cadre de l'exercice de la police des eaux et des milieux aquatiques,

- les services des agences de l'eau au travers du suivi de leurs programmes d'intervention. 
LA LUTTE CONTRE LA POLLUTION ET L'AMÉLIORATION DE LA QUALITÉ DES EAUX

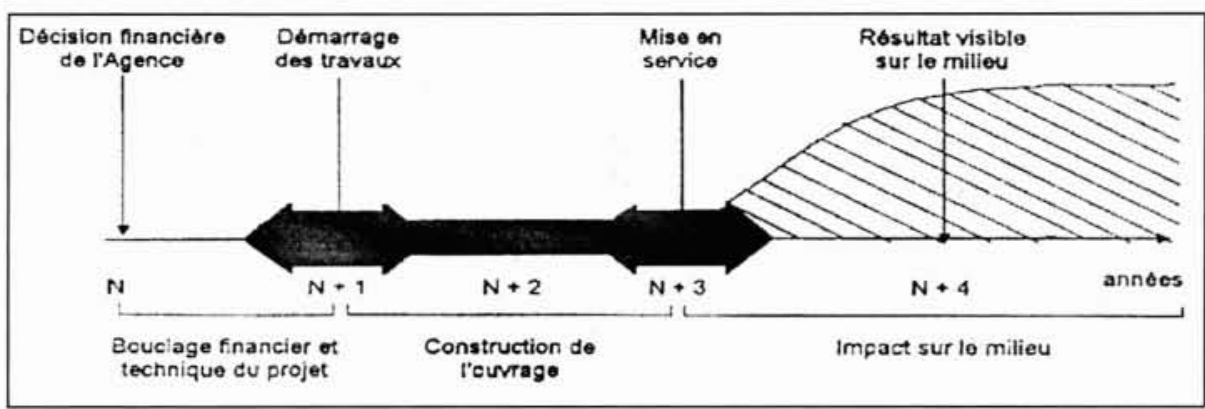

La prise en compte des effets de "différé"
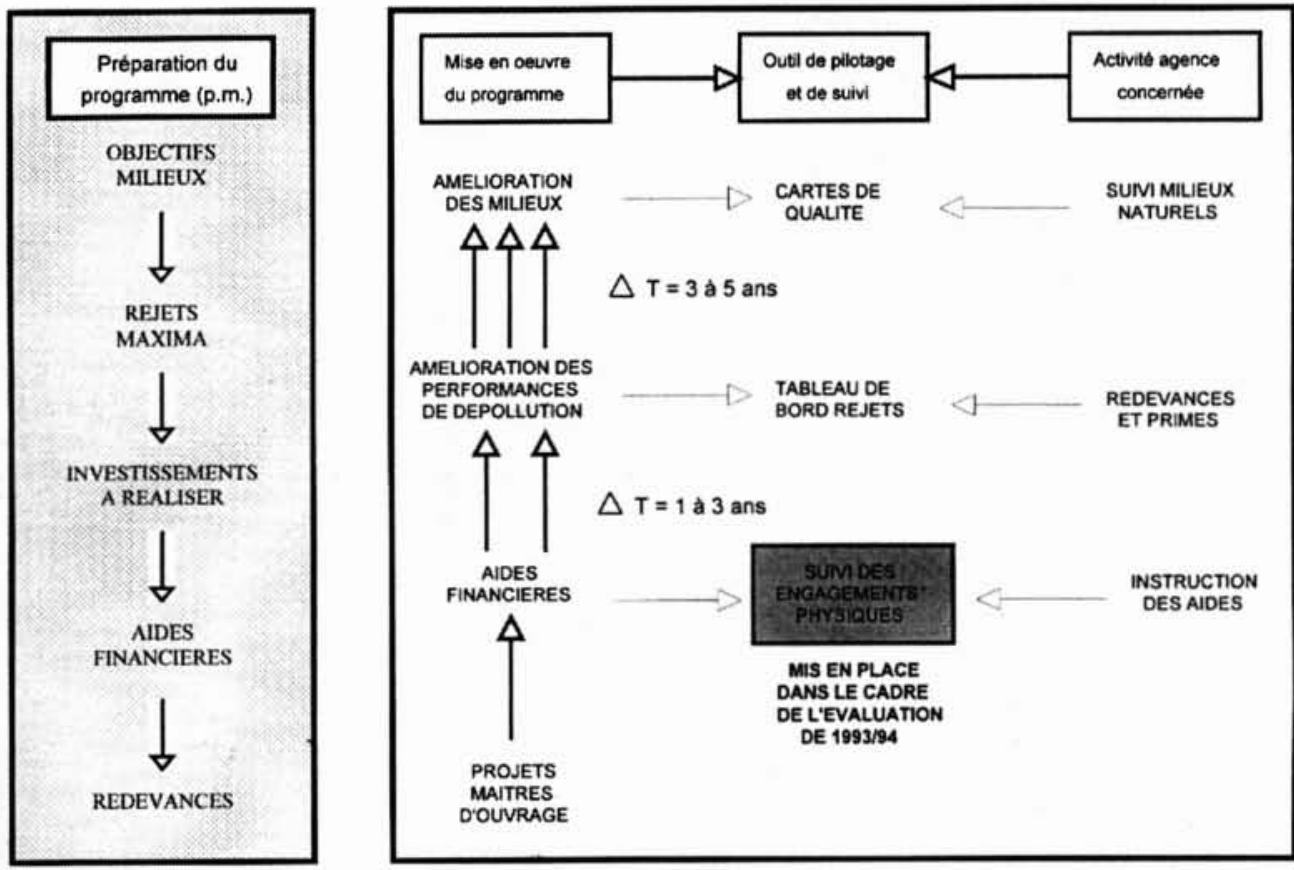

L'architecture du tableau de bord des agences.

Il fera également appel aux outils nationaux existants et en premier lieu le Réseau National des Données sur l'Eau (RNDE) et l'Observatoire National des Zones Humides (ONZH).

\section{II $\square$ LES 7ÈMES PROGRAMMES DES AGENCES DE L'EAU}

Les 7èmes programmes des Agences de l'Eau ont été bâtis parallèlement à l'élaboration et à l'adoption des SDAGE par les Comités de Bassin ; ils en constituent un outil d'application privilégié. Les objectifs principaux de ces programmes, en compatibilité avec ceux définis dans les SDAGE pour les 15 années à venir, s'articulent autour de six priorités :

- l'assainissement-épuration des collectivités locales,

- la lutte contre la pollution des industries,

- la maitrise des pollutions d'origine agricole,

- l'amélioration et la protection de la ressource en eau,

- l'alimentation en eau potable,

- et la préservation des milieux naturels.

Le suivi de ces objectifs nécessitant une évaluation périodique de leur degré de réalisation afin de contrôler l'adéqua- tion entre stratégie, moyens et objectifs, les agences de l'eau ont mis progressivement en place des dispositifs de suivi de leur interventions.

\subsection{Systèmes de suivi}

D'une manière générale ces systèmes de suivi comprennent les trois familles d'indicateurs physiques suivantes (cf. schémas ci-après relatifs à l'assainissement/épuration) :

- suivi de l'état du milieu naturel à l'aide des différents réseaux de mesure regroupés au sein du RNDE,

- évaluation de la réduction des atteintes au milieu naturel à partir des activités redevances et primes,

- détermination des caractéristiques des réalisations engagées dans le cadre des instructions des aides des agences.

Les indicateurs d'état des milieux naturels, dont les valeurs sont connues avec un décalage de plusieurs années, permettent d'évaluer a posteriori l'efficacité de la politique de l'eau (cf. suivi précité des SDAGE).

Venant en complément des indicateurs financiers habituels et ayant comme objectifs d'évaluer les gains attendus des investissements aidés par les agences, les indicateurs physiques de programme, accessibles et renseignables dans le 
cadre des activités de base des agences (instructions des aides, redevances et primes) se décomposent en indicateurs d'actions et indicateurs d'effets.

\subsection{Les indicateurs d'actions}

Cette première famille d'indicateurs permet le suivi des objectifs "d'actions" des programmes des agences de l'eau, c'est-à-dire de réalisation des opérations nécessaires à l'obtention des principaux objectifs "d'effets".

Ils consistent en l'estimation des effets attendus des investissements aidés par les agences de l'eau en relation avec les principaux objectifs de leurs programmes d'intervention : assainissement-épuration des collectivités locales, lutte contre la pollution des activités économiques, aménagement et protection des ressources en eau, préservation des milieux aquatiques et humides, alimentation en eau potable.

Outre le suivi au cours des programmes des niveaux de satisfaction de leurs objectifs, ils permettent également des analyses coûts-efficacités des ouvrages aidés dans le cadre de l'instruction des dossiers d'aide.

Par allégement du dispositif antérieur, afin de le rendre plus pertinent et plus lisible au niveau national, et par adaptation aux inflexions des $7^{\text {èmes }}$ programmes, le dispositif retenu comporte 29 indicateurs.

\subsection{Les indicateurs d'effets}

Cette deuxième famille d'indicateurs permet le suivi des objectifs "d'effets", c'est-à-dire de résultats au bénéfice des usagers et de la préservation du patrimoine naturel aquatique, qui constituent la finalité des actions des agences de l'eau.

Par exploitation et croisements complémentaires ils permettront également le suivi global de l'assainissement des collectivités locales et des industriels (taux de collecte et d'épuration, destination finale des boues des Step), et la confrontation des pollutions éliminées aux investissements de collecte-épuration aidés, ou des pollutions nettes rejetées à l'évolution du milieu naturel.

Ces indicateurs, au nombre de 26 , concernent essentiellement les collectivités locales et les industriels, car il est apparu prématuré d'y intégrer la dépollution des élevages (>70 UGBN) en raison du caractère purement forfaitaire de leurs estimations.

A noter également qu'ils peuvent être complétés par les suivis pluriannuels effectués par les DDASS, des populations alimentées par une eau non conforme (cf. suivi précité des SDAGE).

\section{III — L'ORGANISATION DE LA GESTION CONCERTÉE LOCALE}

Sollicités pour de multiples usages, rivières, nappes et milieux aquatiques continentaux et littoraux peuvent être localement très menacés. Lorsque les efforts consentis in situ pour les préserver n'ont pas suffi à résoudre les conflits et à dégager les conditions d'un partage et d'une gestion équilibrée de la ressource, la loi sur l'eau du 3 janvier 1992 propose une nouvelle démarche de planification locale : le SAGE, schéma d'aménagement et de gestion des eaux.

\section{1 les SAGE}

Ces schémas au-delà des frontières administratives et des oppositions d'intérêt, rassemblent riverains et usagers sur un territoire cohérent autour d'un projet commun : satisfaire les besoins de tous sans porter d'atteinte irréversible à l'environnement.

Avec les SAGE, les élus, usagers, industriels, agriculteurs, gestionnaires d'ouvrage, associations, services de l'Etat, établissements publics, réunis au sein de Commissions Locales de l'Eau (CLE) :

- se concertent pour décider comment réduire les pollutions, éviter l'épuisement des ressources en eau, lutter contre les inondations, restaurer les paysages riverains. Le SAGE est une œuvre collective qui concilie la protection de notre patrimoine "eau" et le développement des activités économiques.

- dans un territoire cohérent le SAGE considère l'eau dans sa globalité. Il est élaboré, non pas à l'échelle d'une portion de rivière, mais à celle d'un bassin versant, d'une vallée et de ses affluents ou d'un aquifère souterrain. Les eaux souterraines, les milieux humides sont pleinement pris en considération dans ces territoires de l'eau.

- et réparent l'oubli : les milieux humides et la végétation des berges, trop souvent maltraités, sont des protecteurs naturels de la rivière, garants de la pérennité de la vie aquatique et des activités qui en dépendent. Ils limitent l'intensité des crues, régularisent les débits et participent à la dépollution.

\subsection{Les Contrats de Rivière (ou de Baie)}

Institués par circulaire du ministre de l'Environnement du 5 février 1981 , les contrats de rivière se sont progressivement ouverts à l'approche globale de la gestion de l'eau et des milieux aquatiques à l'échelle d'un bassin versant. Ils ambitionnent de redonner vie à la rivière à la fois par l'amélioration de la qualité de l'eau, la restauration et l'entretien des berges et du lit, la prévention des crues et la mise en valeur de l'espace rivière. Ils font l'objet d'un mode de concertation élargi et réunissent l'ensemble des acteurs de l'eau au sein de Comités de Rivière (ou de Baie). Ils sont appelés à devenir la traduction opérationnelle privilégiée des SAGE en traduisant leurs objectifs dans des programmes d'aménagement et de gestion privilégiant les méthodes douces et tirant parti des potentialités écologiques des cours d'eau. Les calendriers des programmes se déroulent le plus souvent sur cinq ans, les agences de l'eau, les départements, les régions et l'Etat contribuant fortement à leurs financements.

Ces deux outils complémentaires se relayent sur le terrain : les contrats peuvent découler des SAGE en offrant un cadre adapté à la réalisation de certaines de leurs orientations sur tout ou partie de leur territoire. A l'inverse, les contrats préalablement engagés représentent un premier diagnostic du cours d'eau et un apprentissage de la concertation que les SAGE consolident par la dimension réglementaire qu'ils apportent. Il appartient aux acteurs locaux de choisir la formule qui répond le mieux aux exigences du terrain.

A rappeler également que devant le risque de gaspillages financiers consécutifs à l'absence d'une démarche locale de recherche de cohérence d'ensemble et d'objectifs précis, le ministère de l'Environnement a encouragé dès 1985 la mise 
au point de Contrats pluri-annuels d'agglomération comportant des objectifs physiques de dépollution, des programmes de travaux et des engagements réciproques de financement.

3.3 L'état d'avancement en fin 1997 de ces procédures de planification et de contractualisation locales fait apparaître :

a) pour les SAGE :

- l'un d'entre eux, celui de la Drôme, en phase de mise en œuvre (SAGE élaboré, adopté et approuvé),

- 32 projets en cours d'élaboration (périmètres délimités, CLE constituées),

- 21 projets en phase d'instruction (avec avis des Comités de Bassin concernés pour 14 d'entre eux),

- 29 projets en phase d'émergence (initiatives locales de constitution de dossiers préliminaires en vue de leur transmission aux préfets pour instruction).

b) pour les Contrats de Rivière (ou de Baie) :

- 31 d'entre eux réalisés (contrats achevés),

- 32 en phase d'exécution (dossiers définitifs agréés et contrats signés),

- 38 en phase d'élaboration (dossiers préalables agréés),

- 26 en phase d'émergence.

\section{IV — LES RETOURS D'EXPÉRIENCES}

La montée en puissance de ces procédures et de leurs suivis conduit tout naturellement à les conforter ou à les infléchir pour tenir compte des réalités rencontrées sur le terrain.

C'est ainsi qu'en matière de :

\subsection{Tableau de bord des SDAGE}

Un bilan critique de leur mise en œuvre est en cours sous l'égide du RNDE ; à l'issu de celui-ci, des ajustements et approfondissements de certains indicateurs seront opérés, notamment avec le concours de l'IFEN.

\subsection{Tableau de bord des 7èmes programmes}

La mise en œuvre des indicateurs réseaux d'assainissement faisant apparaître des écarts notables entre les agences de l'eau, un audit en a été confié en 1997 par la Direction de l'eau à un bureau d'études ; cet audit a débouché sur l'établissement d'un guide d'évaluation des indicateurs d'actions des réseaux d'assainissement, destiné aux instructeurs chargés du traitement des demandes d'aide des maîtres d'ouvrage. Son utilisation permettra de faciliter le travail de ces instructeurs tout en améliorant la validité des indicateurs et l'homogénéité de leur mise en œuvre au sein des agences.

\subsection{P Procédure SAGE}

A partir des travaux du colloque du Cercle Français de l'Eau de juin 1997 et du séminaire national SAGE de novembre 1997 sur les premiers retours d'expériences :

- une circulaire du ministre de l'Aménagement du territoire et de l'environnement aux préfets, leur a rappelé l'intérêt de cette procédure et la nécessité de l'accompagner, ou de la susciter en cas de conflits locaux ou de problèmes importants,

- un programme d'actions inter-bassins pour 1998/99 prévoit notamment :

- la mise en place d'un réseau de concepteurs de ces schémas (annuaires, base documentaire, ...),

- une meilleure prise en compte de la dimension paysagère dans ceux-ci,

- l'actualisation du guide méthodologique de leur élaboration.

\subsection{Procédure Contrat de Rivière (ou de Baie)}

Une évaluation de cette politique contractuelle a été demandée par le Directeur de l'Eau aux Conseils généraux des P \& C et du GREF ; celle-ci comprendra notamment : - un bilan critique, par grands bassins, de ces politiques contractuelles au regard des exigences nouvelles de la Loi sur l'Eau.

- une analyse détaillée de la mise en œuvre réelle de ces contrats par rapport aux engagements pris par les différents partenaires et de leurs effets sur l'évolution des milieux et des usages de l'eau,

- des propositions d'améliorations des pratiques d'élaboration, d'approbation et d'application de ces contrats incluant une réflexion sur les modalités envisageables de déconcentration de la procédure d'agrément.

\section{$\mathrm{V} \square$ CONCLUSION}

En conclusion, la gestion concertée et décentralisée de l'eau en France repose donc sur :

- la mise en œuvre des outils de planification, de programmation et de gestion de l'eau institués par les Lois de 1964 et de 1992,

- un suivi à l'aide de tableaux de bord de cette mise en œuvre,

- une évaluation des résultats obtenus et une adaptation en conséquence de ces outils. 